\title{
Per la storica estimazione delle concordanze onomastiche latino-etrusche.
}

\author{
Von E. Lattes.
}

La giusta e benefica reazione contro il corssenianismo, mentre salvò l'ermeneutica etrusca dall'affogare nel ridicolo, insieme di necessità rese incerti gli studiosi eziandio rispetto al fatto certissimo delle concordanze fra l'onomastico etrusco ed il latino; sicchè ora dall'un canto annunzia taluno, come importante per l'apprezzamento di quelle, la scoperta, certamente notevole, di uno o due Tarconi nella Lidia o nella Licia o fra gli Hetei; ora qualche altro si industria a dimostrare, di certo utilmente, come una o due dozzine di nomi propri italici si ritrovino in questa 0 quella terra, a dir cosi, pelasgica, nelle medesime condizioni, parrebbe, che cento e cento analoghi loro, per es. nell' Etruria o nel Lazio; d'altro canto pur coloro che studiarono seriamente il monumentale Zur Geschichte der lateinischen Eigennamen di W. Schulze, tratto tratto ripetono con profonda persuasione che alla fabbrica del comune edificio onomastico i due popoli contribuirono del pari e, come dire, tanto ad un bel circa ciascuno apportò, quanto prese.

Ora il primo fatto che colpisce chi confronta i due onomastici, gli è quello della stragrande quantità di nomi personali che si ripetono inalterati quasi lettera per lettera in entrambi; cosi, se per esempio consideriamo quelli che cominciano per $c$ - $(k-)$, troviamo $\left.{ }^{1}\right)$ etr. Caie Cae C(ae) Caia Caea Cai Kavi Kavia Caaies, lat. Caius Gaius Gavius Gaia Gavia osc. Gaaviis; Caciu Caceis Caceinei, Cacius Cacinus (CIL XI. 6700. 186. a. b.); Cavili Caveli, Cavilius Gavilius; Kaviates Gaviatius; Kavini Cavinei, Cavin(n)ius Gavinius Gavinna; Cazi Cassius (bilingue); Caznei

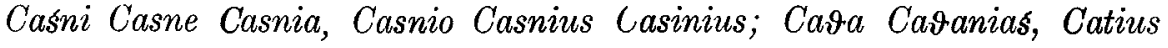

1) V. pei documenti il mio Secondo seguito del Saggio di un indice lessicale etrusco nello Memorie della $R$. Accademia di Archeologia Lettere e Belle Arti di Napoli, 1910, II 182-294, ben s'intende, quasi sempre conforme per i nomi personali a W. Schulze e con le riserve da lui ripetutamente fatte (Lat. Eigenn. 62. 100. 107. 282. 434. ecc.), quanto alla certezza della rispondenza in questo o quel caso ed alla compensazione delle dubitazioni e dei possibili errori, grazie al numero ed alla similarità delle prove per quasi tutte le categorie. 
Catianius; Ka१leś Catulus; Kâuniia Catonia; Ceicnas Cecnas, Caecina; Kaizu Caeso; Caile, Caele Caelius; Cailinal Caelennia; Caipur, Caipor; Kaisie Caisias, Caesius; Calapi Calabius; Calati Calatia; Calerial Galeria; Cale Calia Callia Cali, Galius Gallius Galia Gallia; Calinei, Calinius Gallinius; Caliti Callidius; Calpurniaś Calpurnius; Calunei Calonius Gallonius; Kamaia Camei Camus, Cammius; Camarine, Camarinus Camarenus; Camnas, Camnius Caminius; Campania Campanius; Campes Campius; Camuris Camurius; Camurinal Camurinus; Canzna Kansinaia, Canusinus; Canina Kaninia: Cantini Cantinius; Cape Capius; Capevani Capuan, Capuanus; Capenate Capenatis; Capras Caprius; Capru Kaprnas, Capronius; Cara Caria, Carinus; Karkanas Carcenius; Carcunia Carcna, Carconius Gargonius; Carcusa Gargossa; Carzin Karse Carses, Carso Carsius; Carinì Carinius; Kariunas Carienius; Karmuniš Carminius; Carna Carnius; Carpe; Carpus Carpius; Carpnati Carpnti, Carpinatius; Carpni Carpinius; Carpunies (incerto), Carponius; Cartlia Cartilius; Casa Casia, Casius; Casuntinial Casntinial, Casontonio (Marsi); Caspres Casperius; Caspu Caspo; Caturus, Caturo Caturonius; Catusa, Catussa (Vibia); Caugial Cauthia, Caudius; Caule Caulias, Colins Golius; Caunu Caunius; Caupis Caupius; Caupne Cauponius; Cauri Caurias, Corius; Causine Causlini, Causinius Cosilinum; Casates Cafates, Cafatius; Cecunia Cencunia, Cigonius; Ceztes Cestius; Ceףurna, Ceternius Caetronius; Ceina Cena, Cenius; Ceisi Cesi, Ceisia Cessius Cisius; Ceisinis Cesina, Cesenius Cesennius Caesennius; Cele Celias Celas, Gelius Gellius Cella; Celmnei Celemna; Celsina Celsinius; Celtas Celtus; Cemu Cemunia, Cemonius Gemonius; Cenepnal Cnepni, Cenopennus; Cesu Ceisu Cesunia, Caesonius Ciso Cisso Cisonius Cissonius Cisionius; Ceti Caedius; Cetisnei Caetennius (cf. Arcmsnei Arcumenna, Percumsna Pergomsna Pergonia, Petsna Petinius, Ratumsna Ratumenna, Statsne Statinius); Cvinti Cuinte, Quintius Quinctius; Ciar७is, Ciartiae; Cilnia Cilni Kilnei, Cilnius; kinaś Cinna; Cipirunia Ciprinius; Cire Cirrius; Cisvite Cisuita, Cisuitius; Cisie Cisius; Claie Klae (incerti), Clavius; Claniu Clauniu, Clani (Philarcuri); Clantie Clante, Clandius; Clauce Claucia; Clevsinas, Clusinius Clusinas; Clevsu, Cleusius Clusius; Cleustes Cleuste Cleusti, Clustius; Clumnei Colominaeus; Clute Cla(u)dia; Cnaives Cnaeve Cneve, Gnaivod Cnaeus; Comlniai Cumlnei Cumni, Cominius; Craca Cracial, Gracius Graceus; Cracina Gracinius; Cravzaguras (cf.

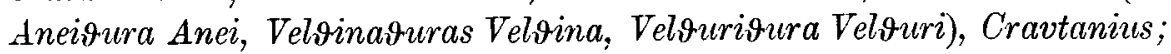
Cranes Granius; Cranpe Cramponius; Craufa Craupania, cf. Corifanius; Creice, Craecius Graecius; Cresa Gresius; Crespe Crispius; Crespini Crispinia; Crisu, Crisius Grisius; Crucu Crocius; Crusi, Grusius; Cuclnies, Cuculnius Coculnius; Cuies, Coius Covius; Cuizlania Cuislania, Cuslanus; Culni, Colona Cullonius; Cupes Cupi, Cupius; Cuprna, Cubrenus Cupri- 
nius; Cupuna Cupna Cupnei, Coponius Copponius Cuppenius Cupiennus Chpanius; Curania Curunas, Coronius; Curce, Gurges Gurgus; Cure Curia, Curius Corius; Curve Kurvenas, Curvius Curvinius o Kurpenas, Corpennius; Cursnis Corsinius; Cusis Cusius; Cusiges Considius (cf. Pes9e

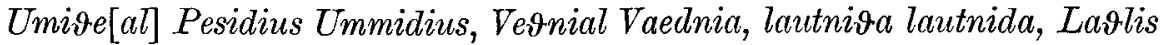
Ladile, Sasnal Sadnal); Cusine Cusinius; Kusiunas Cusnia, Cusonius Cosonius; Cuspi Cvspi, Cuspius; Cusu Quso; Cutu Cutius; Cutuna Cutnas, Cotonia Cutenius. - E cosí via nello stesso modo per tutte le altre lettere dell'alfabeto ${ }^{1}$ ).

Al primo, s'aggiunge poi un seconde fatto non meno singolare e capitale: la diffusione progressiva in Roma, nel Lazio, in Italia ed in tutto l'orbe romano, come luminosamente documentò W. Schulze, dei nomi propri derivati coll' "etruskischte" (Herbig Idg. Forsch. 1909 XXVI 369) dei suffissi, cioè col suffisso -na, sia tale quale in -ena -enna, sia latinizzato in -enius -ennius e simili. Così per esempio Abenna Abennius Abinnius, Adenna Adinius, Alina Alinna Alinnius, Arcumenna, Artenna Artinius, Avena Avennius, Aulinna Aulinius, Catinna Catinnius, Herenna Herinna, Largenna Largennius, Laucinna, Papsenna, Ratumenna (R)atumennia, Spurinna Spurinnia, Tapsenna, Thormena, Velcenna Volcinius, Velina Velenius, Verginna Verginius, Vetina Vetinius, Vibenna Vibinnius Vivennius, Volasenna, Volusenna Volusenus; ed altresi per es. Corona Coronius, Cotena (falisco) Cutennins, Patina, Perna Perennius, Poena Poenius Foenius, Saenae Saenius, Tarcna Tarquenna Tarconius, Tarna Taronius; ed ancora per es. Mortennius, Aminnius Aminius Amnius, Anquirinnius, Arsinius, Audenins Ausenius, Caesennius, Essennius, Faltennius, Flavennius, Sociennius; cf. etr. Apinal, AvЭenna (inc.), Alnial, Arcsmnei, Artinial, Aunaś Avines, Aulni, Catnis Caףnis, Herina Larcna, Laveina, Papsinas, Ratumsna (ef. sup. Cetisnei), Spurina Spurienas, Tapsina, Turmna, Vel $\chi(n)$ a Vel $\chi$ inei, Velinei, Velini, Varena, Vetna, Vetinei, Vipinas, Velasnei, Velusina; ed altresì ef. Xurunal, Cutnas, Patnei, Perna,

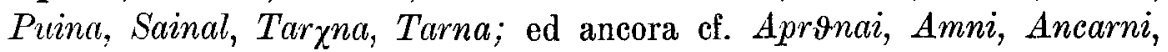
Arzni, Augnal, Usinies, Cesina, Ezna, Tlavienas, Suenei ${ }^{2}$ ).

1) Cosi quanto ai nomi personali, comincianti per a- (v. Saggio di un indice lessicale etrusco e Seguito del Saggio ecc. nelle Memorie della R. Accademia di Archeologia eec. di Napo ii, 1908. 1909, I 3-78 e 79-179), per es. Acari Ancari, Ancarius; Acilu Acilius; Akius, Accius; Aclani Aclni, Aclenus; Acries, Acrius Agrius; Acranis Aernis, Agranius; Acsi Azsi Ahsi Asi, Axius; Avini Avinivs; Avulni Avolenus, Aulni Aulinna, Aunial Aunius; Alu Aluni, Alonius, ecc. Notevolissimi fra tutti di questa lettera sono Aremsnas Arcmsnei Aroumenna e Arnour Arrunturius, come sup. Cnepni Cenepnal, Cenopennus.

2) Tanto più rimarchevoli tornano i -na ecc. latinoetruschi, in quanto dall' indagine dell' Herbig Idg. Forsch. cit. 367-380 risulta che nelle etrusche epigrafi più si scende coi tempi e più scemano i nomi personali appunto in -na e simili 
Un terzo fatto analogo al secondo, ma ancor più sorprendente, anch'esso in parte per la prima volta lumeggiato da W. Schulze, offrono la progressiva diffusione ed il romanizzamento di alquanti nomi personali etruschi formati mediante suffissi assai più remoti dall'uso latino, ehe non -na -enna -ennius, e nell' Etruria medesima assai meno frequenti e persino sconosciuti fuor di certi territori. Tale per es. il suffisso -sa, quasi ignoto a Perugia a quasi proprio soltanto della regione chiusina, suffisso rappresentato a Roma (Saggi e Apunti 173, ef. W. Schulze 326) in tempi già bene antichi da P. Cornelius Calussa primo pontefice massimo plebeo (Liv. XXV 5): cf. lat. Calusius, lat. etr. CIE 1235 C. Sentius C. F. Grania natus Hannossa con etr. 4867 L(ar)ף Seiante Hanusa Helial clan, etr. lat. Pabassa Gargossa per etr. Papasa Carcusa (inc.) etr. Presntessa Presntesa e Cauślinissa Cauślinisa. Qui spettano quindi eziandio, fra l'altro, lat. Namusa Tarcussa Pandúsa e notevolissimo CIL III 5352 Vibia Catussa, dove il -sa etrusco, attestato dal pretto etrusco Vibia, confluì verisimilmente col $-s a$ illirico e macedone di Clagissa Voltisa e simili, passato ai Greci verisimilmente in $\beta a \sigma \iota \lambda i \sigma \sigma a$ (W. Schulze 40 n.) ed a noi in ,contessa duchessa ${ }^{1}$ ). Analogamente il suffisso -aea femminile del maschile - $a$ rappresentato da lat. etr. Pabaea A. l. Rufina coliberta e moglie, pare, di Paba A. l. Philomusus CIL XI. 2777 Volsinii); suffisso verisimilmente volgare nato in tempi recenti dall'arcaico -aia di etr. Kansinaia Kamaia Hirminaia femm. di Canzna Cama-s Hermena-s. $\mathrm{E}$ così altri non pochi (-tor -torius -tra-tru-tronius ecc.), secondo le luminose dimostrazioni di W. Schulze.

All'acume erudito del quale dobbiamo poi la scoperta di un quarto singolarissimo fatto, vale a dire la regolare parallela evoluzione in ambo gli onomastici del fenomeno delle ampliazioni sinonime, per effeto del quale ad esempio nelle bilingui latino etrusche rispondono a lat. Alf-iuts Arr-iu-s Var-ia natus, etr. Alf-n-i Arnt-n-i Var-n-al senza variazione di significato ampliati mediante il suffisso nasale. Ora, come per figura lat. Afinius Aferius, Babrinius Baberius, Buceinius Buccerius, Casinius Caserius, Caspennius Casperius, Egenia Egeria, Gabinius Gaberius, Labinius Laberius, Latinius Laterius, Numenius Numerius, ecc. cosi etr. Arsina Arsira, Aruśni Aruseri, Cepeni Cipiru, Cupana Cupures,

e piủ crescono quelli in $-a-i e-e$ e simili, conforme al progresso della romanizzazione.

1) Come accanto a $\beta c \sigma \iota \lambda i \sigma \sigma \alpha$ sta $\beta \alpha \sigma \iota \lambda i v v \alpha$ (cf. lat. gallina regina Agrippina ecc.), cosi di contro a lat. Calussa Namusa lat. etr. Hannossa etr. Hanusa stanno etr. Calunei Namonias Hanunia; cosi pure etr. Pabassa Papasa e Papania, Viliasa e Viliania, ecc. salvochè per lo più in etrusco il derivato nasale funge da femminile del derivato sibilante, perchè questo fu ambigenere (cf. etr. msc. Aulesa Aulza, femm. Velisa Veliza ed i -sa maritali naturalmente femminili), laddove quello apparisce sopratutto femminile (cf. W. Schulze $323 \mathrm{sg}$.) 
Papni Paperis; sicchè non torna illecito sospettare che analoga relazione possa per avventura intercedere pur fra gli appellativi capeni caperi, spureni spureri, nungene nungeri, alla maniera, se mai, che lat. campanius camparius, extraneus extrarius e simili. Tali anzi appariscono in siffatto riguardo la regolarità ed il parallelismo dei due onomastici, da potersene integrare, sì la serie con $n$, e sì quella con $r$, quando con l'aiuto dell'uno e quando dell'altro, e porre per atto di esempio a riscontro di etr. Eizenes $\Theta$ amries Lauteri lat. Aeserius Daminius Lautinius. E così via per altre serie numerose, benchè di rado integre da ambo le parti.

Serbai per ultimo un quinto fatto, ehe, dopo il primo, a me sembra di gran lunga il più ragguardevole: vale a dire la perfetta uguaglianza della formole onomastiche etrusche normali alle normali latine, le une e le altre ugualmente contradistinte dalla invenzione e dalla prevalenza assoluta del prenome. In effetto, prenomi veri, quali oggi ancora per eredità romana intendiamo, possedettero nell' Italia medesima, insieme coi Latini Falischi Umbri Oschi ed altrettali, i soli Etruschi, e non i Messapi od i Veneti, e non in Italia o fuori i Greci, i Galli, i Germani, nè, direi, qual sia si altra gente ariana od anariana: il che dimostra che presso gli Etruschi, come presso i Latini e loro affini, le vicende istoriche ingenerarono quelle condizionni sociali e quegli instituti famigliari, da cui spontaneamente di necessità conseguì la funzione per alquanti nomi personali, comuni più o meno a tutte le genti e famiglie, di distinguere per entro ad esse ed allo Stato i gentili uno dall' altro; nomi singolari, abbreviati di regola e preposti al nome comune dei gentili di ciascuna gente e famiglia, come appunto, insieme coi Romani e loro affini, usarono gli Etruschi, entrambi fra loro concordi, e diversi dagli altri tutti, eziandio nel normale collocamente davanti al nome e nella scrittura abbreviata, che noi del pari ereditammo insieme con lo stesso prenome ${ }^{1}$ ).

1) Cf. Saggi e Appunti intorno all' isc. etr. della Mummia 182 sg. n. 132 ed $A$ che punto siamo con la questione della lingua etrusca (Atene $e$ Roma 1910 XIII. 310-312). Già negli arcaici epitaffi di Orvieto il prenome vedesi ben distinto dal nome, ma si scrive aricora sempre distesamente: mi Venelus Spurienas, mi Venilus TveYelies, mi Mamarces Vel9ienas, mi Mamarces Papalnas; per contro già ne' più antichi testi latini il prenome apparisce negli ingenui certi (e peró non in Novios Plautios o Tibis Pilipis o Retus Gabinio C. s. e simili) abbreviato. Niente altro che aggettivi prenominali si trovano essere d'altronde i più dei nomi latini ed etruschi (cf. da ultimo W. Schulze Lat. Eigenn. 65. 262 e Borghesi ib. 243); si direbbe quindi che dapprima in tempi preistorici l'onomastico etrusco $\theta$ latino constó di meri prenomi, corne nelle steppe della Russia, dove oggi ancora il discorso comune si limita a quelli (per es. Alexis Alexandrovitch, Anna Pavlovna, quello un Karenine, questa una Scherer), e nella segregazione dei Ghetti, prima che la legge prescrivesse il nome di famiglia, quantunque ivi non siasi trattato per verità propriamente di prenomi, ma di nomi biblici divenuti poi tali. Il sistema prenominale schietto parrebbe anzi rispondere alla persuasione 
Oltre poi al prenome fu peculiare degli Etruschi, come dei Latini ed affini, la formola onomastica prenominale trimembre, stata dapprima prenominale bimembre e cresciuta poi, specie presso gli Etruschi ed i Latini, sino a diventare sovente per lo meno quadrimembre ${ }^{1}$ ).

Ora, in presenza di fatti cotanto cospicui per volume e qualità, i due o tre Tarconi asiatici e la dozzina doppia o tripla dei nomi personali e locali latino-etruschi riscontrati fuori d' Italia più 0 meno precisamente ${ }^{2}$ ), benchè assai degni, e questi e quelli, d' attenzione, mi sembrano promettere per la questione etrusca solo quel tanto che simili rapporti diedero sempre per altrettali questioni, dove con l' aiuto insidioso della toponomastica torna tanto facile confondere e tanto difficile distinguere: invero, nella povertà del mio ingegno, io non so immaginare che gli Etruschi avendo avuto con alcuni popoli d' Italia relazioni cotanto strette e varie ed efficaci e diffuse, possano averne tenuto con altri affatto diversi di simili e per qualità e quantità paragonabili con quelle attestate dall' onomastico latino-etrusco, pienamente conformi alla tradizione circa la storia della coltura paleoitalica. D'altro canto, certo per la medesima povertà mia intellettuale, non so affatto intendere come mai alla costruzione di un edificio tanto regolare ed omogeneo, quanto l'onomastico predetto, abbiano potuto contribuire insieme concordi due popoli, se mai, onninamente differenti nella lingua, quali si reputano per la testimonianza indeclinabile, nell' ignoranza nostra dei testi non onomastici, gli Etruschi ed i Latini; e ciò senza che appaia possibile segnare con sicurezza, sul fondamento di fatti evidenti, piuttosto che di argomentazioni subiettive, la linea di separazione fra il contributo degli uni e quello degli altri $\left.{ }^{3}\right)$.

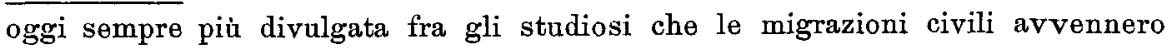
per piccoli e piccolissimi aggruppamenti; dai prenomi dei loro capi o padri poteronsi poi ricavare i nomi con cui si addimandarono le genti da essi procreate nelle nuove terre. Quanto al collocamento, ricordo che soltanto nell' Etruria meridionale il prenome spesso posponesi al nome, $\theta$ che presso gli Umbri il prenome patronimico segue immediatamente quello della persona ricordata prima del suo nome, come oggi ancora presso i Russi.

1) V. Le formole onomastiche dell' epigrafia etrusca nelle Memorie del R. Istituto Lombardo 1910 XIII. 64 sg.

2) Cf. da ultimo Kannegiesser, Klio 1910 XI. 26-47, dove naturalmente i nomi confrontati concordano, per lo più, soltanto nella base; inoltre, non meno naturalmente, ben di rado egli potè eseguire il suo giusto divisamento (p. 27) di tener conto anzitutto dei nomi occorrenti in einer alten Inschrift etruskischer Sprache', laddove di necessità dovette egli profittare quasi sempre dei testi chiusini e perugini, tutti più o meno recenti, e lasciar da parte gli arcaici orvietani, fiesolani, volterrani, aretini e cortonesi.

3) Secondo I. B. Carter, Röm. Mitteil., $1910 \mathrm{XXV} 78$ spariscono le difficolta, quando 'man sich die Weise ihrer (d. h. der Etrusker) ersten Ansiedelungen klar macht', cioè 'nicht als Eroberer', 'in friedlicher Weise und in kleineren 
La contradizione, quale a noi apparisce, insanabile fra la lingua di essi testi e quella dell' onomastico, risulta poi essere tanto più flagrante, in quanto che da una parte le mutuazioni dei Latini (-enna -ennius -sa, ecc.) sono ben certe, laddove quelle che si attribuiseono agli Etruschi si fondano appunto sull' enimmatica diversità della lingua dell' onomastico da quella, quale a noi tuttodi sembra, delle bende di Agram, della tavola fittile capuana, del piombo di Magliano, del cippo di Perngia ed altrettali contesti; e dall' altra parte tutto ciò che nell' onomastico dicesi etrusco esclusivamente, in realtà è soltanto peculiare dell' uso etrusco, ma non apparisce punto contrario al resto e tanto meno in sè $\mathrm{e}$ per sè, sotto qualsiasi riguardo, anariano ${ }^{3}$ ).

Gruppen', i quali verosimilmente 'gar keinen Widerstand fanden' e 'durch ihre Kultur überlegen' seppero 'sich mit den Einheimischen auf freundlichen Fuß zu stellen', sicchè 'allmählich erfolgte die Verschmelzung' e con questa 'die Stärke': qui ancora peró sgraziatamente ('es mag eine Schwäche meiner Denkweise sein', secondo disse di sè Skutsch, Glotta 1908 I. 320) debbo confessare di non comprendere affatto, come mai codesti piccoli gruppi stranieri abbiano potuto insegnare pacificamente agli inculti indigeni alloglossi la religione di Giove ottimo massimo e della triade capitolina (p. 80 sg.), la limitazione (p. 81. 84), l'aruspicina (p. 85 sg. e v. tantosto altra n.) e diventare 'die Amme der kleinen Göttin Rom' (p. 88). Se alcun poco di siffatti miracoli mi fosse dato capire, capirei certo insieme come mai, fra l'altro, i civili maestri stranieri abbiano gettato a mare i più dei loro nomi personali, contro l'uso delle genti più barbare, per surrogarvi quelli dei loro barbari alloglossi scolari. Pertanto logicamente, a parer mio, ritorno in sostanza W. Schulze alla dottrina della mutuazione italica, propugnata da Pauli e Schaefer (cf. Bugge, Bezz. Beitr XI 45) affermando (Lat. Eigenn. 64), che 'ein großer Teil des Materials einen doppelten Entlehnungsprozeß durchgemacht hat, zuerst aus dem Lateinischen oder Umbrischen ins Etrukische, dann zurück aus dem Etruskischen ins Lateinische', e che (ib. 263, cf. Herbig Idg. Forsch. 1903 XXVI 275) 'die Etrusker, die allerorten mitten, unter, neben oder über einer lateinischer Bevölkerung saßen, auch eine große Anzahl fertiger Gentilnamen von ihren Nachbaren oder Vorgängern im Besitze des Landes übernommen'; alle gravi difficoltà (cf. Saggi e Appunti 182 n. 132) della quale mutuazione, volle appunto ovviare la pensata della cooperazione, e meglio, speriamo, un di o l'altro ovvierà con la sua geniale erudizione il medesimo W. Schulze. D'altra parte non meno logicamente, ma, per me, con maggiore probabilità, affermó Edoardo Meyer Gesch. des Aliert. II. 703 senz'altro che 'ein sehr großer Teil der römischen Eigennamen ist etriskisch'.

1) Cosi per es. in sè e per sè i prenomi di esclusivo uso etrusco $\nabla e l$ Oanqvil sembrano, se mai, equivalenti a lat. Herius (cf. amb. heri per lat. vel) ed a. lat. etr. Minerva (cf. lat. mens e tongere osc. tanginúd esc.); cosi pure Arn 9 , se mai, tanto più facilmente in sè e per sè potrebbe interpreviarsi 'aratore' in quanto che solamente assai di rado se ne incontra il femminile. Cosi in sè e per sè il suffisso -na, vero 'Leitfossil' (Skutsch) geografico (cf. Herbig, Idg. Forsch. cit. 374) come il ligure -asco ed il preellenico -vףos; del quale il Conway, The prehellenic Inscriptions of Praesos 154, confessò appunto di essere 'never been able to see any ground', perchè si tenesse indizio che la 'Urbevölkerung' la quale se ne serviva fosse 'non-Indo-European'. Bensi Herbig op. cit. 375. n. 1 distingue 
Forse però codesta contradizione trova nell' Italia stessa, ed anzi, se mai, ben vicino all' Etruria, un esiguo riscontro nelle discrepanze, nè poche, nè piccole, dell' osco dall' umbro e di entrambi dal latino, in onta alle tante e tanto grandi somiglianze dei due e dei tre idiomi fra loro; esiguo riscontro che, se mai, si allargherebbe alle infinite discrepanze delle lingue indoeuropee in mezzo a tanta comunione di basi e auffissi e forme, e riceverebbe forse lume dalla causa cui soglionsi quelle per molta parte attribuire, cioè la reazione delle favelle parlate dai precedenti abitatori. Per gli Etruschi, nuova spia di siffatte reazioni etniche darebbe oggi per avventura anche il filone, testè avvertito, delle somiglianze lituslave ${ }^{1}$ ): per essi appunto però l' analogia, già scarsa, verrà in ogni caso ancor più diminuita da ciò, che per l'osco e l'umbro ed in genere per le nostre parlate, il quesito o già si affaccia sotto il solo riguardo linguistico, o può per lo meno limitarsi ad esso; laddove per gli Etruschi esso si complica con altri quesiti di varia origine, non senza nuova contradizione apparente o vera; giacchè mentre l'onomastico etrusco ci tiene legati all' Italia ed anzi al Lazio, certe tradizioni greche, insieme coi fegati di Piacenza, Volterra e Babilonia ${ }^{2}$ ) e con la pietra preellenica

finemente l'etr. femm. Petrunai (peligno Ptruna) dall' 'aus dem Lat. ins Etr. zurückübersetzte petruni (Petronius)': né io nego che questa possa essere la verita, ma mi trattiene ancora da credere che debba tenersi tale, il fatto dello aversi cento e mille esemplari analoghi, e della suprema improbabilità, direi, che il caso cento e mille volte siasi divertito a mettere nella bocca di due popoli, reputati differentissimi per la lingua e la provenienza, parole siffatamente simili da confondersi. Per mia parte a tutte le difficolta sopra discorse, non tengo che la risposta 'ignoramus'; e confesso preferirla alle proposte spiegazioni, le quali a me, colpa di certo la veduta corta di una spanna, finora nulla spiegano.

1) Alludo ad etr. $l \chi$, lituano lyka got. libi nei numerali, in sè e per sè, ben s'intende, senz' alcuna relazione con 'dieci'; a etr. e slav. -na -sa -ia -ca femminili; a $-l$ etrusco slavo ed armeno; al prenome prevalente nella serie onomastica si presso gli Etruschi, $\theta$ si presso gli slavi ecc. secondo accennai Rendic. Ist. Lomb. 1910 pag. 276-282 'Di alcune vere od apparenti somiglianze fra la lingua etrusca e le lituslave' (cf. Endzelin Glotta III 275).

2) Secondo F. B. Carter (n. 8) p. 85 sg. "hat unsere Kenntnis der etruskischen Haruspizin die allergröBten Fortschritte gemacht, und zwar durch Erforschung des entsprechenden babylonischen Systems': sgraziatamente rinvia egli per le prove 'auf die Arbeiten Morris Jastrows' e 'besonders' ai Proceedings of the American Philosophical Society (Philadelphia) Bd. 47 S. 105-129, S. 646 676'; dove nel primo luogo tutt' altri parla di storia naturale e matematica, e nel secondo appena ricordansi qua e lì gli Etruschi con la scorta del Thulin e di G. Körte a proposito della 'Hepatoscopy and Astrology in Babylon and Assyria', di cui soltanto il Jastrow si occupa; a suo avviso (p. 655) il fegato di Piacenza "dating from.about the third century B. C. was used as an object lesson for instruction in hepatoskopy, precisely as the clay model of a liver dating from the 
di Lenno e con le molteplici indicazioni dell' archeologia, ci fanno guardare all' Oriente.

Checchè sia di ciò, concludo osservando che l'estimazione storica dell' onomastico latino etrusco costringe bensì, nel parer mio, a formulare la questione della lingua etrusca alquanto più crudamente che non si soglia, ed a riconoscere anche nell' onomastico l'assoluta prevalenza degli Etruschi, come quelli dei quali soli sappiamo con certezza che diedero certe maniere di nomi, e possiamo fondatamente presumere che introdussero il prenome e fissarono la formola onomastica; insieme però essa ci dà modo di ricavare dalle difficoltà stesse e dalle contradizioni del problema insegnamenti profittevoli per l'ermeneutica, e però atti a prepaiarne ed affrettarne la soluzione. $\mathrm{E}$ primieramente in generale dalle cose predette riceve conferma splendidissima la sentenza del Bücheler (Rhein. Mus. XXXIX 409) doversi considerare come una ipotesi per sè stessa poco attendibile, che due potenti tronchi linguistici come l'Etrusco ed il Latino, pur non avendo avuto tra loro una innata comunanza, fossero vissuti per tanti secoli l'uno accanto all' altro senza che esercitassero tra di loro una considerevole influenza ${ }^{1}$ ); e però si conferma insieme doversi inevitabilmente contenere, come nel vocabolario latino osco umbro e simili, così nei testi etruschi di ogni maniera numerosi elementi latini 0 simili, comunque e quando che sia venuti, non di rado verisimilmente più 0 meno velati, che giova anzitutto ricercare per farne leva nelle interpretazioni, senza suggestioni corsseniane, come senza anticorsseniane preoccupazioni, e senza limitazioni aprioristiche di qualità, perchè nessun limite di per sè stesso avvertesi nelle lingue rispetto ai vicende-

Hammurabi period was used in a Babylonian temple school'. Crede poi il Carter, 'daB die Etrusker diesen Teil ihrer Religion wahrscheinlich in einer ausgebildeten Form nach Italien mit sich brachten': ora io mi domando come mai salvo lat. haru- di cui dubitavasi che provenga da un cuneiforme har 'fegato' (cf. Skutsch, La lingua etr. trad. Pontrandolfi p. 33), tutti i termini tecnici del Lazio, di cui ci pervenne notizia (Saggi e App. 179 fissum familiare od hostile o vitale, praesegmina, rimae e rupturae, limes, labes, fibrae ecc.) sono prette latine? E mi permetto osservare d'occasione, che anche il rin vio (p. 81 n. 5) a 'Schulze bei Thulin' per lat. gruma da gr. $\gamma \nu \tilde{\omega} \mu \alpha$ per via etritsca, come 'unzweideutiges Zeugnis' dell' etrusca origine della limitazione, sfortunatamente non serve, giacchè ivi si ripete soltanto, come da un pezzo sappiamo, che Schulze 'annunziò' la esposizione e dimostrazione futura di quel suo giusto pensiero etimologico (io addurrei a rincalzo Cnepni Crepni, cntram cletram cltral) con riferimento evidente all' indice della nota memoria berlinese (Ber. Berl. Akad. 1905 XXVI 709).

1) Cosi a proposito di lat. frontesia rimpetto ad etr. frontac col quale inclino anch'io pur sempre a mandare eziandio l'osco frunter, cioè frunt-er (cf. Deecke Etr. Forsch. VII 57 e Planta Gramm. II 640 frun-ter, esitante a cagione di -ter per -tor) come front-ac, anche per confronto, se mai, con etr. caper-c allato a caperi cape. 
voli influssi ed alle mutuazioni ${ }^{1}$ ): ricercare intendo, con la somma cautela che il 'vestigia terrent' insegna a chi dagli erramenti compassionevoli del passato trae norma per le indagini future. In particolare poi, la storica spregiudicata estimazione delle concordanze onomastiche latino-etrusche permette di aggiungere ai fatti positivi alquanti negativi di uguale e persino maggiore importanza. Io non so cioè persuadermi (cf. W. Schulze 420 tat. Vatia vatia e Sura sura) che per es. i nomi personali etruschi. Cucuma, Cumere, Kurtinas, Curtines e così per esempio Ćnares, Cramnal, finora senza preciso riscontro, pare, nell' onomastico nostro latino, non rendano probabile sino ad un certo punto che gli Etruschi possedettero od adottarono gli appellativi latini cucuma, cumerus, cortina, e così gnarus gramina; similmente Tlavienas già in un arcaico epitaffio di Orvieto e कlave a Volterra, mi paiono dare sino ad un certo punto indizio che pur gli Etruschi dissero per avventura flavus; sicchè quindi per esempio nell' etr. Prasin [a] e lat. etr. Prasna io m'attenterei di

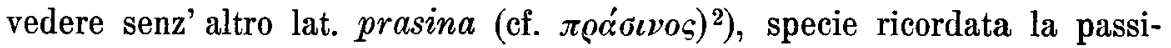
one degli Etruschi per le corse de' cocchi e dei cavalli (Müller-Deecke, Die Etrusker I. 222. 370, cf. II 271 'Tomba delle Bighe').

Milano.

1) Cf. Wackernagel Sprachtausch und Sprachmischung nelle Nachr. d. Ges. d. Wiss. Göttingen 1904 II $90 \mathrm{sgg}$. e Skutsch La lingua etr. tr. it. 46. 66. 70; bene però Herbig 1dg. Forsch. cit. 361 ammonisce contro l'abuso del 'Lehngut'.

2) W. Schulze, Zur Gesch. d. lat. Eigenn. 91 confrontò lat. Prastina; giustamente in ogni caso prescinde egli dal 'num si recte lectum sit dubito' del Panli CIE 424 circa l'aretino Prasin[a], forse dimentico in quel momento del chinsino 2592 aprasnar ravmatrii, ossia A(ulus) Prasna Ar(untis filius) Rav(entia) matre d'alfabeto latino e di lingua semietrusca. 